\title{
Constitutive androstane receptor promoted- hepatomegaly and liver regeneration is partially via yes-associated protein activation
}

\author{
Huichang $\mathrm{BI}^{1}$, Yue $\mathrm{Gao}^{2}$, Shicheng $\mathrm{Fan}^{2}$, Hua $\mathrm{Li}^{3}$, Yiming Jiang ${ }^{1}$, Xinpeng Yao ${ }^{1}$, Shuguang \\ Zhu $^{3}$, Xiao Yang ${ }^{1}$, Ruimin Wang ${ }^{1}$, Jianing Tian ${ }^{1}$, Frank Gonzalez ${ }^{4}$, and Min Huang ${ }^{5}$ \\ ${ }^{1}$ School of Pharmaceutical Sciences, Sun Yat-sen University \\ ${ }^{2}$ School of Pharmaceutical Sciences, Sun Yat-sen University \\ ${ }^{3}$ Department of Hepatic Surgery, the Third Affiliated Hospital, Sun Yat-Sen University \\ ${ }^{4}$ National Cancer Institute, National Institutes of Health \\ ${ }^{5}$ Institute of Clinical Pharmacology
}

April 29, 2020

\begin{abstract}
Background \& Purpose: The constitutive androstane receptor (CAR) belongs to nuclear receptor superfamily. The administration of CAR agonist TCPOBOP to mice leads to hepatomegaly but the mechanism is unclear. Yes-associated protein (YAP) is a downstream factor of Hippo signaling pathway, which is a potent regulator of organ size and tissue homeostasis. This study examined the role of YAP in CAR-promoted hepatomegaly and liver regeneration. Experimental Approach: The effect of CAR on liver enlargement and liver regeneration was evaluated in wild-type (WT) mice, liver-specific YAP-deficient mice, and partial hepatectomy (PHx) mice. KI67 and CTNNB1 staining were performed to evaluate the proliferation response and hepatocytes size. The protein levels of YAP and its downstream targets were measured and Co-IP was conducted to explore the protein-protein interaction between CAR and YAP. Key Results: The results suggested TCPOBOP increases the liver/body weight ratio in WT mice and PHx mice. Hepatocytes enlargement occurred around the central vein area, while the number of KI67+ cells increased around portal vein area. The translocation of YAP was induced and its downstream targets were upregulated after CAR activation via TCPOBOP. Co-IP results revealed a potential protein-protein interaction between CAR and YAP. However, CAR-induced hepatomegaly was still observed in Yap-/- mice. Conclusion and Implications: CAR activation promotes hepatomegaly and liver regeneration in part by inducing nuclear translocation of YAP and interaction with YAP pathway, which provides new insights for understanding the physiological functions of CAR, and suggests the potential for manipulation of liver size.
\end{abstract}

\section{Introduction}

The constitutive androstane receptor (CAR, NR3I1) is a xenobiotic-sensing nuclear receptor and a member of the nuclear receptor superfamily (Konno, Negishi \& Kodama, 2008). Like other nuclear receptors, CAR is composed of a DNA-binding domain, a large carboxy-terminal ligand-binding domain (LBD), and a poorly conserved amino-terminal domain (Willson \& Kliewer, 2002). CAR is localized in the cytoplasm by forming a complex with HSP90 and the cytoplasmic CAR retention protein (CCRP). Once activated, CAR dissociates from the cytoplasmic complex and is translocated into the nucleus where it forms a heterodimer with retinoid $\mathrm{X}$ receptor alpha $(\mathrm{RXR} \alpha, \mathrm{NP} 2 \mathrm{~B} 1)$ and activates transcription of its target genes (Kodama \& Negishi, 2006).

CAR activates transcription of its target genes encoding various enzymes and proteins involved in drug metabolism and transport when exposure to xenobiotics (Konno, Negishi \& Kodama, 2008; Wei, Zhang, 
Egan-Hafley, Liang \& Moore, 2000). In addition to xenobiotics, CAR can also regulate the elimination of toxic endobiotics such as bilirubin and bile acids, so one major function of CAR is detoxification (Eloranta \& Kullak-Ublick, 2005; Sugatani et al., 2002). However, recent studies have also revealed a key role for CAR in various physiological and pathophysiological processes occurring in the liver including gluconeogenesis, metabolism of fatty acids, metabolism of lipids, hormonal regulation, proliferation of hepatocytes, and hepatocarcinogenesis (Kachaylo, Pustylnyak, Lyakhovich \& Gulyaeva, 2011). CAR is also involved in metabolic diseases including jaundice, cholestasis, thyroid homeostasis and obesity (Dash et al., 2014; Qatanani \& Moore, 2005).

TCPOBOP (1,4-Bis [2-(3,5-dichloropyridyloxy)] benzene), a strong agonist of CAR, was reported to induce robust hepatocyte proliferation and hepatomegaly in mice livers (Costa, Kalinchenko, Tan \& Wang, 2005). Moreover, the rapid proliferation response induced by TCPOBOP can protect liver from failure even after massive tissue loss by $91 \%$ hepatectomy (Tschuor et al., 2016). The mechanism of TCPOBOP-induced hepatomegaly was studied in previous reports. C-Myc and FOXM1 mediated proliferative programs are key mediators of TCPOBOP-CAR induced direct liver hyperplasia (Blanco-Bose et al., 2008). $\beta$-catenin (CTNNB1) deficiency decreases the impact of CAR activation on hepatocytes proliferation (Ganzenberg, Singh \& Braeuning, 2013). Disruption of EGFR and MET signaling leads to a dramatic impairment of the TCPOBOP-induced proliferative response without altering CAR activation (Bhushan et al., 2019).

Yes-associated protein (YAP) is a key downstream factor of Hippo signaling pathway, which is a potent regulator of organ size and tissue homeostasis (Kowalik et al., 2011). When Hippo signaling is ON, YAP is phosphorylated by LATS1/2 and binding with 14-3-3 in cytoplasm. When Hippo signaling is OFF, YAP can be dephosphorylated and translocated into nucleus where it binds with TEAD to activate transcription of genes involved in cell survival, growth, and proliferation (Patel, Camargo \& Yimlamai, 2017). According to previous studies, YAP increases organ size and causes aberrant tissue expansion in mice. YAP activation reversibly increases liver size more than 4-fold (Camargo et al., 2007). Removal ofMst1 and Mst2 (the upstream of YAP) in liver, which activates YAP, results in significant liver enlargement and dysplasia (Song et al., 2010). In addition, YAP also participates in liver regeneration after partial hepatectomy (PHx). Hepatocyte nuclear accumulation of YAP increased in parallel with hepatocyte proliferative activity after PHx. Deleting Yap from hepatocytes blocked the nuclear accumulation of pSmad2 and EMT-like response, as well as their proliferation (Oh, Swiderska-Syn, Jewell, Premont \& Diehl, 2018). Inhibition of MST1 and MST2 augments liver repair and regeneration by activating the downstream effector YAP and promoting cell growth (Fan et al., 2016).

Most recently, YAP/TEAD activation was found to participate in CAR-dependent proliferation of murine hepatocytes (Abe et al., 2018). However, this study used an in vitro cell-based system and an animal experiment of verteporfin (an inhibitor of YAP/TEAD interaction) treatment. The mechanistic influence of CAR activation on YAP signaling has not been clarified and the relationship between CAR and YAP remains unknown. Therefore, the current study aims to elucidate the role of YAP in CAR-induced hepatomegaly using liver-specific YAP-deficient mice, to investigate whether YAP is associated with CAR-promoted liver regeneration, and further explore the relationship between CAR and YAP.

\section{Methods}

\section{Animal experiment}

Male C57BL/6J mice (8- to 9-week-old) were obtained from Laboratory Animal Center of Sun Yat-sen University (Guangzhou, China). Liver-specific YAP-deficient mice and paired WT mice were generated by Shanghai Model organisms Center, Inc (Shanghai, China). Male C57BL/6J mice were i.p. injected with vehicle (Corn oil, Aladdin, C116025) or $3 \mathrm{mg} / \mathrm{kg} / \mathrm{d}$ TCPOBOP (Sigma-Aldrich, T1443, St. Louis, MO). Liver tissue and serum samples were harvested after 5 and 10 days. WT mice werei.p. injected with vehicle or $3 \mathrm{mg} / \mathrm{kg} / \mathrm{d}$ TCPOBOP following PHx. Tissue and serum samples were collected at 2 and 5 days after 
the surgery. Male WT and Yap ${ }^{-/-}$mice (8- to 9-week-old) were i.p. injected with vehicle (Corn oil) or 3 $\mathrm{mg} / \mathrm{kg} / \mathrm{d}$ TCPOBOP and the tissue samples were collected at day 10. Serum and liver samples were snap frozen in liquid nitrogen, then stored at -80 for further use. A portion of liver was immediately fixed in 10\% formalin for histological section. The animal treatments were approved by the Institutional Animal Care and Use Committee at Sun Yat-sen University, Guangzhou, China. The entire study stuck to the 3Rs principle of animal experiments, under the guideline of ARRIVE guidelines with respect.

\section{Histological and biochemical assessment}

According to our previous publication (Jiang et al., 2019), liver samples were fixed in formalin, embedded in paraffin, sectioned and stained for haematoxylin and eosin (H\&E staining). Paraffin-embedded sections were stained with $\beta$-catenin antibody (BD Biosciences, 610153, San Jose, CA) and KI67 antibody (Abcam, ab15580). Olympus BX41 microscope was used to examine the stained liver sections. Serum alanine aminotransferase (ALT), aspartate transaminase (AST), alkaline phosphatase (ALP), total bile acid (TBA) and total bilirubin (TBLI) levels were detected by commercially available kits (Nanjing Jiancheng Bioengineering Institute, China) guided by the manufacturer's protocols.

\section{Quantitative real-time PCR analysis}

Total RNA from liver tissue samples were extracted by using Trizol reagent. Single-strand cDNA was synthesized by reverse transcription of total RNA using Primer Script RT reagent Kit (Takara, Japan). All sequence of primers used for quantitative RNA analysis were listed in Supplementary information. The mRNAs were amplified in Biosystems 7500 Real-time PCR reaction system using SYBR Premix Ex-Taq II Kit (Takara, Japan) according to the manufactory's instructions. The fold changes of mRNA levels were analyzed by $\delta \delta \mathrm{Ct}$ method.

\section{Western blot analysis}

Total proteins from liver tissue samples were extracted by using the RIPA lysis buffer containing $1 \% 100$ mM PMSF then the concentrations were determined by BCA Protein Assay Kit (Thermo Scientific, 23227, Rockford, IL). Protein levels were detected by Western bolt analysis. Protein samples of $40 \mu \mathrm{g}$ per lane were separated by a 10\% SDS-PAGE gel and transferred onto polyvinylidene fluoride membranes. After blocking with $5 \%$ non-fat milk for $1 \mathrm{~h}$, the blots were incubated with different antibodies overnight at 4 , followed by secondary anti-rabbit (Cell Signaling Technology, \#7077, Danvers, MA)or anti-mouse antibodies (Cell Signaling Technology, \#7076, Danvers, MA) at room temperature for $1 \mathrm{~h}$ on the next day. The ECL Detection Kit (Engreen Biosystem, Beijing, China) was employed to develop the blots. The intensity of the protein bands was assessed by Quantity One software (Bio-Rad, California, USA).

\section{Co-immunoprecipitation (Co-IP)}

HepG2 cells (ATCC, Virginia, USA) were cultured in DMEM containing 10 \% FBS. Co-immunoprecipitation (Co-IP) was performed using Thermo Scientific Pierce Co-IP kit (Thermo Scientific, 26149, Rockford, IL) following the manufacturer's protocol as described in our previous report (Jiang et al., 2019). Samples were analyzed by western blotting using anti-IgG (Abcam, ab133470), anti-CAR (Abcam, ab62590) and anti-YAP (Cell Signaling Technology, \#14074, Danvers, MA).

\section{Co-localization assay}

According to previous publications (Jiang et al., 2019), HepG2 cells (ATCC, Virginia, USA) were cultured in DMEM containing 10\% FBS. Cells were treated with DMSO or $10 \mu \mathrm{M}$ CITCO (Sigma-Aldrich, C6240, St. Louis, MO) for $48 \mathrm{~h}$. Following incubation, cells were fixed in $4 \%$ paraformaldehyde for 30 min and $0.5 \%$ triton $\mathrm{X}-100$ for $10 \mathrm{~min}$ at room temperature. Then, cells incubated with rabbit polyclonal anti-CAR (Abcam, ab62590) and mouse monoclonal anti-YAP (R\&D Systems, MAB8094, MN, USA) overnight at $4^{\circ} \mathrm{C}$. Cells were stained with secondary antibodies including anti-mouse IgG Alexa Fluor 488 (Cell Signaling Technology, \#4408, MA, USA) and anti-rabbit IgG Alexa Fluor 647 (Cell Signaling Technology, \#4414, MA, USA). Images were acquired using a confocal microscope (Olympus FV3000, Japan). 


\section{Materials}

Antibodies used in this study include anti- $\beta$-catenin (BD Biosciences, 610153), anti-KI67 (Abcam, ab15580), anti-YAP (Cell Signaling Technology, \#14074), anti-CTGF (Sangon biotech, D260212), anti-CYR61 (Cell Signaling Technology, \#39382), anti-ANKRD1 (Sangon biotech, D121628), anti-CCNA1 (Sangon biotech, D220507), anti-CCND1 (Sangon biotech, D220509), anti-CCNE1 (Sangon biotech, D151593), anti-p-YAP Ser127 (Cell Signaling Technology, \#13008), anti- $\beta$-actin (Cell Signaling Technology, \#8457), anti-Lamin B (Cell Signaling Technology, \#13435), anti-C-Myc (Cell Signaling Technology, \#9402), anti-CTNNB1 (Sangon biotech, D199519), anti-MET (Sangon biotech, D160981) and anti-FOXM1 (Proteintech, 13147-1-AP).

\section{Statistical Analysis}

All values were presented as the means \pm standard deviation (mean \pm S.D.). Two-tailed Student's $t$ tests were used to assess the differences between groups using SPSS 23.0 software (IBM Analytics, USA) and GraphPad Prism 7.0 software (GraphPad Software, USA). Differences with $P$ values $<0.05$ were considered significant.

\section{Results}

\section{CAR activation induces hepatomegaly in mice liver.}

To evaluate the effect of hepatomegaly induced by CAR, mice were treated for 5 or 10 days with the CAR specific agonist TCPOBOP (Figure 1A). Liver/body weight ratios were significantly increased after treated by TCPOBOP (Figure 1B). Liver morphology also showed an obvious enlargement both 5 and 10 days after TCPOBOP treatment (Figure 1C). H\&E staining did not reveal significant liver injury (Figure 1D). Serum ALT, AST and ALP were also examined and there was no significant difference between the TCPOBOP group and vehicle group (Figure S1A), suggesting that the hepatomegaly was not induced by liver injury.

It was reported that TCPOBOP can induce hepatocyte proliferation and the increase hepatocyte cell size (Blanco-Bose et al., 2008). To characterize the size of hepatocytes, CTNNB1 staining was performed and an increase of cell size around the central vein $(\mathrm{CV})$ area was observed (Figure 1E and $1 \mathrm{~F}$ ), while there was no significant change in cell size around the portal vein (PV) area (Figure S1B). Expression of KI67 was determined by immunohistochemistry to assess hepatocyte proliferation. The number of $\mathrm{KI}_{6} 7^{+}$cells around the PV area was dramatically increased after 5 or 10 days of TCPOBOP treatment, indicating a high level of cell proliferation induced by CAR (Figure $1 \mathrm{G}$ and $1 \mathrm{H}$ ). However, the KI67+ cells did not appear around the CV area (Figure S1C).

\section{CAR activation promotes liver regeneration after partial hepatectomy in mice.}

To explore whether CAR activation can promote liver regeneration, a 2/3 hepatectomy model was used and treated with TCPOBOP (Figure 2A). A significant increase of liver size and liver/body weight ratio were observed in TCPOBOP treated group (Figure 2B and 2C).

H\&E staining revealed no liver injury occurred (Figure 2D). Serum ALT, AST, TBLI and TBA didn't changed significantly after TCPOBOP treatment, indicating normal liver function (Figure S2A). CTNNB1 and KI67 staining were also performed. Hepatocytes around the CV area showed a dramatic enlargement (Figure 2E, quantification shown in Figure 2F) which was absent around the PV area (Figure S2B). The number of $\mathrm{KI}_{6} 7^{+}$cells were apparently increased around the PV area after treatment with TCPOBOP (Figure $2 \mathrm{G}$ and $2 \mathrm{H}$ ) while there was no expression around the $\mathrm{CV}$ area (Figure $\mathrm{S} 2 \mathrm{C}$ ). These data suggest that CAR activation promotes hepatocyte enlargement around the CV area and proliferation around the PV area, which further facilitates liver regeneration.

YAP pathway is involved in CAR-induced hepatomegaly and liver regeneration. 
YAP is a potent regulator of organ size and tissue homeostasis (Kowalik et al., 2011). The role of YAP/TEAD in CAR-induced hepatomegaly was also investigated. The protein levels of total YAP and its downstream targets CTGF, ANKRD1 and CYR61 were measured in hepatomegaly and liver regeneration models. All of these proteins were upregulated in TCPOBOP-treated mice, especially at Day 10 (Figure 3A). The proliferation-related proteins CCNA1, CCND1 and CCNE1 were all significantly upregulated after TCPOBOP treatment. The upregulation of nuclear YAP and the downregulation of cytoplasmic p-YAP suggested that the YAP pathway was activated in this process (Figure S3A).

In the PHx mice model, the protein levels of YAP and its downstream targets ANKRD1 and CYR61 were upregulated, while CTGF showed a slight increase in TCPOBOP-treated mice. The proliferation-related proteins CCNA1, CCND1 and CCNE1 were all significantly upregulated after TCPOBOP treatment, especially at Day 2 after PHx (Figure 3B). Similarly, the nuclear YAP and the cytoplasmic p-YAP showed the same trend as the hepatomegaly model, which indicated that the YAP pathway was activated in these processes (Figure S3B).

Nuclear translocation is essential both for CAR and YAP to activate their downstream targets, thus the possible co-localization of CAR and YAP was examined. HepG2 cells were treated with hCAR selective agonist CITCO. The result suggested that the CAR activation enhanced translocation of YAP (Figure 3C). Co-immunoprecipitation (Co-IP) experiment was then performed to confirm the potential protein-protein interaction between CAR and YAP and a clear interaction between CAR and YAP was observed (Figure $3 \mathrm{D})$.

\section{CAR-induced hepatomegaly is partially due to YAP signaling.}

To further explore the role of YAP in TCPOBOP-induced hepatomegaly, Yap ${ }^{-/}$mice were used and treated with TCPOBOP for 10 days (Figure 4A). The liver/body weight ratio still showed an increase in TCPOBOP treated $\mathrm{Yap}^{-/-}$mice, but decreased compared with TCPOBOP-treated WT mice, which suggested that YAP is only partially involved in CAR activation-induced hepatomegaly (Figure 4B). A slight shrinkage of liver was noted in Yap ${ }^{-/-}$mice in the treated group compared with the counterpart in WT mice (Figure 4C).

Serum ALT, AST and ALP indicated no significant difference between the treatment and vehicle group (Figure S4A). The YAP protein and its downstream targets together with proliferation-related proteins were also measured (Figure S4B and S4C). YAP was totally depleted in Yap ${ }^{-/-}$mice, YAP downstream targets CTGF, ANKRD1 and proliferation-related protein CCND1 were upregulated in TCPOBOP-treated group both in WT and Yap ${ }^{-/-}$mice.

Immunohistochemical staining of CTNNB1 and KI67 was conducted revealing that hepatocyte size in the $\mathrm{CV}$ area was still enlarged (Figure $4 \mathrm{E}$ and $4 \mathrm{~F}$ ), while the number of $\mathrm{KI}_{67}^{+}$cells was increased in the PV area in TCPOBOP-treated Yap $^{-/-}$mice (Figure $4 \mathrm{G}$ and $4 \mathrm{H}$ ), but the effect was weaker compared with TCPOBOP-treated WT mice. CTNNB1 staining in the PV area and KI67 staining in the CV area are shown in Figure S4D and S4E.

\section{Other factors are involved in CAR-induced hepatomegaly.}

To investigate other possible factors involved in CAR-induced liver enlargement, the mRNA levels of other related genes such as C-myc, Foxm1, Ctnnb1, Egfr and Metwere measured in TCPOBOP-treated WT mice and $\mathrm{Yap}^{-/-}$mice (Figure 5A and 5B). The results suggested that C-myc, Foxm1, Ctnnb1 and Metwere significantly upregulated in TCPOBOP-treated WT mice and TCPOBOP-treated Yap $^{-/-}$mice. Then the protein levels of C-Myc, FOXM1, CTNNB1 and MET were measured and the protein levels of C-Myc, CTNNB1 and MET were significantly upregulated in TCPOBOP-treated WT mice and Yap ${ }^{-/-}$mice, indicating that these signaling pathways also contribute to CAR-induced liver enlargement when $Y a p$ was absent (Figure 5C and 5D). These data suggested that many different pathways were involved in CAR -induced hepatomegaly, the knockout of Yap only relieve the liver enlargement to some extent but cannot totally eliminate the proliferation effect caused by other pathways such as C-Myc, CTNNB1 and MET signaling. 


\section{Discussion and Conclusions}

CAR plays an important role in the control of both endogenous and xenobiotic compound metabolism. It was reported that CAR activation by TCPOBOP leads to hepatomegaly and an increase in both hepatocyte growth and proliferation in mice. Since YAP is a potent regulator of liver size and tissue homeostasis, its role in the hepatoproliferative effects of CAR was investigated. Most recently, YAP/TEAD activation was found to play a role in CAR-dependent proliferation of murine hepatocytes (Abe et al., 2018). However, the role of YAP in CAR-induced hepatomegaly and liver regeneration, and the relationship between CAR and YAP remains unknown. In the current study, CAR activation significantly promoted nuclear translocation of YAP and directly interacted with YAP, which consequently promotes hepatomegaly and liver regeneration thus indicating a potential role for YAP in CAR-induced hepatomegaly and liver regeneration. These findings provide new insights for understanding the physiological functions of CAR.

It was reported that CAR activation via TCPOBOP can induce robust hepatocyte proliferation and hepatomegaly in mouse livers and promote liver regeneration after liver tissue loss (Costa, Kalinchenko, Tan \& Wang, 2005; Tschuor et al., 2016). In the current study, CAR-induced hepatomegaly and liver regeneration was confirmed in WT and the PHx mouse model. All mice were male in order to eliminate the gender difference, since CAR can be activated by estrogens, although to a lesser degree than by exogenous ligands such as TCPOBOP (Kachaylo, Pustylnyak, Lyakhovich \& Gulyaeva, 2011; Min, Kim, Bae, Petz \& Kemper, 2002). CAR-induced hepatomegaly and liver regeneration in this study is mainly caused by hepatocyte enlargement and proliferation. Hepatocytes proliferation can be observed only in the PV area, while the increase of cell size was only shown in the CV area. A possible reason for this phenomenon is that CAR activation induces the expression of enzymes and perhaps the enlargement of subcellular structures, since enzymes related to glycogen synthesis, glycolysis, lipogenesis, ketogenesis, and detoxication are preferentially situated in the CV area (Jiang et al., 2019; Jungermann \& Katz, 1989).

YAP is a key downstream factor of the Hippo signaling pathway, which is a crucial regulator of organ size and tissue homeostasis (Kowalik et al., 2011). A previous study demonstrated that another nuclear receptor, pregnane $\mathrm{X}$ receptor (PXR), can also induce liver enlargement and promote liver regeneration via activation of YAP (Jiang et al., 2019). Both PXR and CAR belong to the superfamily of nuclear receptors (Chai, Zeng \& Xie, 2013), suggesting that CAR may activate or interact YAP as well. A previous cell-based study suggested that there is a functional crosstalk between CAR and YAP in the nucleus of hepatocytes, but there is no evidence suggesting a functional relationship between CAR and YAP (Abe et al., 2018). In this study, CAR activation can significantly promote the nuclear translocation of YAP and then upregulate the downstream targets of YAP. Furthermore, Co-IP assays showed a potential protein-protein interaction between CAR and YAP, which suggest the important role of YAP in CAR-induced liver enlargement and regeneration. However, additional evidence such as surface plasmon resonance and isothermal titration calorimetry is needed to confirm their interactions, and to determine the amino acids that lead to the CAR and YAP interaction.

Yap liver-specific knockout mice revealed that CAR-induced hepatomegaly was only partially dependent on the YAP pathway, suggesting other regulatory factors may be involved in CAR-induced liver enlargement. Many regulators were reported to be involved in CAR activation-induced hepatomegaly such as C-Myc, FOXM1, CTNNB1, EGFR and MET. According to previous reports, the activation of CAR can still promote hepatomegaly in Ctnnb1 knockout mice (Ganzenberg, Singh \& Braeuning, 2013). The depletion of C-Myc also only partially restored the enlargement of liver (Blanco-Bose et al., 2008). Similarly, liver enlargement can still be observed in EGFRi+MET double knockout mice after treatment with TCPOBOP for 10 days (Bhushan et al., 2019). These studies indicated complicated mechanisms in CAR-induced liver enlargement and regeneration process. In the current study, the mRNA and protein levels of related factors involved in hepatocytes enlargement and proliferation were measured. In WT mice, the expression of C-Myc and MET were significantly upregulated after treated by TCPOBOP. Interestingly, the level of these proteins were even higher in TCPOBOP-treated Yap ${ }^{/-}$mice than WT mice. We hypothesize that these proteins were upregulated to a higher extent to compensate the proliferative response reduced by the loss of Yap . 
CTNNB1 was not significantly changed consistent with a previous report (Ganzenberg, Singh \& Braeuning, 2013), but can be downregulated in $\mathrm{Yap}^{-/-}$mice, and then be induced by the activation of CAR, suggesting that there may be a crosstalk between the CTNNB1 and YAP pathways. On the other hand, a previous study revealed that PXR can also promote hepatomegaly and liver regeneration via YAP pathway. However, the activation of PXR failed to induce liver enlargement and liver cell fate change when YAP was depleted, indicating that PXR-induced hepatomegaly is totally in a YAP dependent manner (Jiang et al., 2019).

YAP downstream targets such as CTGF, ANKRD1 were upregulated even in Yap $^{-/-}$mice. We hypothesize that these proteins might be regulated by other factors simultaneously. CTGF is a member of the CCN family, which is involved in diverse biological processes such as cell adhesion, proliferation, and angiogenesis. Previous studies reported that CTGF can be regulated by the Wnt/ $\beta$-catenin signaling pathway (Deng et al., 2007; Li et al., 2012). Meanwhile, it is also a potential target of Wnt and BMP signaling (Luo et al., 2004). ANKRD1 is a potent regulator of early cardiac development, it can be significantly upregulated in cardiac hypertrophy and heart failure (Kojic et al., 2010). ANKRD1 was cooperatively induced by both the TGF- $\beta$ and Wnt pathways in normal murine mammary gland epithelial cells. The upregulation of ANKRD1 can be observed in models of Wnt/ $\beta$-catenin-induced tumors (Labbe et al., 2007). Our results suggested that the $\beta$-catenin signaling pathway was activated in $\mathrm{Yap}^{-/-}$mice, and thus CTGF and ANKRD1 were still significantly upregulated in $\mathrm{Yap}^{-/-}$mice.

Cyclin D and cyclin E are two major classes of cyclins expressed in mammalian cells during the G1 phase of the cell cycle (Geng et al., 1999). Protein levels of CCND1 and CCNE1 were upregulated significantly at both time points in TCPOBOP-treated WT mice. This suggested that CAR activation induces hepatocyte proliferation by promoting G1-S transition. Since DNA synthesis in hepatocytes was terminated 4-5 days after PHx, the liver mass was restored so the proliferative response was much weaker at Day 5 (Jiang et al., 2019). Thus, in the PHx vehicle group, the expression of CCNE1 was downregulated at Day 5 while CCND1 didn't change significantly.

Liver possesses the ability to regenerate in response to injury. Liver regeneration can be defined as compensatory hyperplasia with the remaining liver tissue expands to meet the metabolic needs of the organism, however, the expanding liver does not regain its original gross anatomical structure. The pathways involved in liver regeneration include cytokines, growth factors, and metabolic networks. The most completely studied model is that of liver regeneration following partial hepatectomy, which 3 of 5 liver lobes $(2 / 3$ of the liver mass) is removed (Mao, Glorioso \& Nyberg, 2014). It was reported that CAR deficiency impaired liver regeneration, while the CAR activation through pharmacological means is sufficient to prevent or rescue the experimental SFSS (small-for-size-syndrome), which is a modified version of extended hepatectomy in mice (eHx, 86\% removed) (Tschuor et al., 2016). The present study showed that YAP, a critical factor of liver regeneration, was activated in TCPOBOP-promoted liver regeneration in PHx model, which aids the liver to regain its normal functions.

In summary, the current study demonstrate that CAR activation can promote liver enlargement and liver regeneration partially by inducing nuclear translocation of YAP and interaction with the YAP signalling pathway. These findings provide new insights for understanding the physiological functions of CAR and suggests the potential for manipulation of liver size. CAR can be a potential target to promote liver regeneration after liver surgery and rescue SFSS during the transplantation of liver. The investigation of protein-protein interaction between CAR and YAP may provide therapeutic strategy that can avoid the side effect of CAR activation and promote its clinical use in the future.

Acknowledgements: The work was supported by the Natural Science Foundation of China (Grants: 81973392), the National Key Research and Development Program (Grant: 2017YFE0109900), the Natural Science Foundation of Guangdong (Grant: 2017A030311018), the 111 project (Grant: B16047), the Key Laboratory Foundation of Guangdong Province (Grant: 2017B030314030), the Local Innovative and Research Teams Project of Guangdong Pearl River Talents Program (2017BT01Y093), and the National Engineering and Technology Research Center for New drug Druggability Evaluation (Seed Program of Guangdong Province, 2017B090903004). 
Conflict of interest: There are no competing interests.

Author contribution: H.B. and M.H. conceived the project. Y.G., S.F., X.Y., R.W., and J.T. performed the experiments. H.L. and S.Z. contributed to the animal models. Y.J., X.Y., and F.J.G participated in the scientific discussion and research design.

\section{REFERENCES}

Abe T, Amaike Y, Shizu R, Takahashi M, Kano M, Hosaka T, et al.(2018). Role of YAP Activation in Nuclear Receptor CAR-Mediated Proliferation of Mouse Hepatocytes. Toxicol Sci 165: 408-419.

Bhushan B, Stoops JW, Mars WM, Orr A, Bowen WC, Paranjpe S, et al. (2019). TCPOBOP-Induced Hepatomegaly and Hepatocyte Proliferation are Attenuated by Combined Disruption of MET and EGFR Signaling. Hepatology 69: 1702-1718.

Blanco-Bose WE, Murphy MJ, Ehninger A, Offner S, Dubey C, Huang W, et al. (2008). C-Myc and its target FoxM1 are critical downstream effectors of constitutive androstane receptor (CAR) mediated direct liver hyperplasia. Hepatology 48: 1302-1311.

Camargo FD, Gokhale S, Johnnidis JB, Fu D, Bell GW, Jaenisch R, et al. (2007). YAP1 increases organ size and expands undifferentiated progenitor cells. Curr Biol 17: 2054-2060.

Chai X, Zeng S, \& Xie W (2013). Nuclear receptors PXR and CAR: implications for drug metabolism regulation, pharmacogenomics and beyond. Expert Opin Drug Metab Toxicol 9: 253-266.

Costa RH, Kalinchenko VV, Tan YJ, \& Wang IC (2005). The CAR nuclear receptor and hepatocyte proliferation. Hepatology 42:1004-1008.

Dash AK, Yende AS, Kumar S, Singh SK, Kotiya D, Rana M, et al.(2014). The Constitutive Androstane Receptor (CAR): a nuclear receptor in health and disease. J Endocrinol\&Reprod 18: 59-74.

Deng YZ, Chen PP, Wang Y, Yin D, Koeffler HP, Li BJ, et al.(2007). Connective tissue growth factor is overexpressed in esophageal squamous cell carcinoma and promotes tumorigenicity through beta-catenin-Tcell factor/Lef signaling. J Biol Chem 282:36571-36581.

Eloranta JJ, \& Kullak-Ublick GA (2005). Coordinate transcriptional regulation of bile acid homeostasis and drug metabolism. Arch Biochem Biophys 433: 397-412.

Fan F, He Z, Kong L-L, Chen Q, Yuan Q, Zhang S, et al. (2016). Pharmacological targeting of kinases MST1 and MST2 augments tissue repair and regeneration. Sci Transl Med 8.

Ganzenberg K, Singh Y, \& Braeuning A (2013). The time point of beta-catenin knockout in hepatocytes determines their response to xenobiotic activation of the constitutive androstane receptor. Toxicology 308: 113-121.

Geng Y, Whoriskey W, Park MY, Bronson RT, Medema RH, Li TS, et al. (1999). Rescue of cyclin D1 deficiency by knockin cyclin E. Cell 97: 767-777.

Jiang Y, Feng D, Ma X, Fan S, Gao Y, Fu K, et al. (2019). Pregnane X Receptor Regulates Liver Size and Liver Cell Fate by Yes-Associated Protein Activation in Mice. Hepatology 69:343-358.

Jungermann K, \& Katz N (1989). Functional specialization of different hepatocyte populations. Physiol Rev 69: 708-764.

Kachaylo EM, Pustylnyak VO, Lyakhovich VV, \& Gulyaeva LF (2011). Constitutive androstane receptor (CAR) is a xenosensor and target for therapy. Biochemistry (Mosc) 76: 1087-1097.

Kodama S, \& Negishi M (2006). Phenobarbital confers its diverse effects by activating the orphan nuclear receptor car. Drug Metab Rev 38: 75-87. 
Kojic S, Nestorovic A, Rakicevic L, Belgrano A, Stankovic M, Divac A, et al. (2010). A novel role for cardiac ankyrin repeat protein Ankrd1/CARP as a co-activator of the p53 tumor suppressor protein. Arch Biochem Biophys 502: 60-67.

Konno Y, Negishi M, \& Kodama S (2008). The roles of nuclear receptors CAR and PXR in hepatic energy metabolism. Drug Metab Pharmacok 23: 8-13.

Kowalik MA, Saliba C, Pibiri M, Perra A, Ledda-Columbano GM, Sarotto I, et al. (2011). Yes-associated protein regulation of adaptive liver enlargement and hepatocellular carcinoma development in mice. Hepatology 53: 2086-2096.

Labbe E, Lock L, Letamendia A, Gorska AE, Gryfe R, Gallinger S, et al. (2007). Transcriptional cooperation between the transforming growth factor-beta and wnt pathways in mammary and intestinal tumorigenesis. Cancer Res 67: 75-84.

Li ZQ, Ding W, Sun SJ, Li J, Pan J, Zhao C, et al. (2012). Cyr61/CCN1 Is Regulated by Wnt/beta-Catenin Signaling and Plays an Important Role in the Progression of Hepatocellular Carcinoma. Plos One 7.

Luo Q, Kang Q, Si WK, Jiang W, Park JK, Peng Y, et al. (2004). Connective tissue growth factor (CTGF) is regulated by Wnt and bone morphogenetic proteins signaling in osteoblast differentiation of mesenchymal stem cells. J Biol Chem 279: 55958-55968.

Mao SA, Glorioso JM, \& Nyberg SL (2014). Liver regeneration. Transl Res 163: 352-362.

Min G, Kim H, Bae Y, Petz L, \& Kemper JK (2002). Inhibitory cross-talk between estrogen receptor (ER) and constitutively activated androstane receptor (CAR). CAR inhibits ER-mediated signaling pathway by squelching p160 coactivators. J Biol Chem 277: 34626-34633.

Oh SH, Swiderska-Syn M, Jewell ML, Premont RT, \& Diehl AM (2018). Liver regeneration requires Yap1TGFbeta-dependent epithelial-mesenchymal transition in hepatocytes. J Hepatol 69: 359-367.

Patel SH, Camargo FD, \& Yimlamai D (2017). Hippo Signaling in the Liver Regulates Organ Size, Cell Fate, and Carcinogenesis. Gastroenterology 152: 533-545.

Qatanani M, \& Moore DD (2005). CAR, the continuously advancing receptor, in drug metabolism and disease. Curr Drug Metab 6:329-339.

Song H, Mak KK, Topol L, Yun K, Hu J, Garrett L, et al. (2010). Mammalian Mst1 and Mst2 kinases play essential roles in organ size control and tumor suppression. Proc Natl Acad Sci U S A 107:1431-1436.

Sugatani J, Yamakawa K, Yoshinari K, Machida T, Takagi H, Mori M, et al. (2002). Identification of a defect in the UGT1A1 gene promoter and its association with hyperbilirubinemia. Biochem Bioph Res Co 292: 492-497.

Tschuor C, Kachaylo E, Limani P, Raptis DA, Linecker M, Tian Y, et al. (2016). Constitutive androstane receptor (Car)-driven regeneration protects liver from failure following tissue loss. J Hepatol 65: 66-74.

Wei P, Zhang J, Egan-Hafley M, Liang SG, \& Moore DD (2000). The nuclear receptor CAR mediates specific xenobiotic induction of drug metabolism. Nature 407: 920-923.

Willson TM, \& Kliewer SA (2002). PXR, CAR and drug metabolism. Nat Rev Drug Discov 1: 259-266.

\section{Figure Legends}

FIGURE 1 CAR activation induces hepatomegaly and increase the liver-to-body weight ratio. (A) Mice were treated by TCPOBOP for 5 days or 10 days. (B) The liver-to-body weight ratio significantly increased in TCPOBOP-treated group ( $n=5$ per group). (C) Liver morphology also showed a liver hypertrophy. (D) H\&E staining suggested that there was no liver injury ( $n=5$ per group). Scale bar $=50 \mu \mathrm{m}$. (E) CTNNB1 
staining was performed to measure the cell size, which indicated an increase of cell size around CV area $(n$ $=5$ per group). Scale bar $=50 \mu \mathrm{m}$. (F) Quantification of CTNNB1 staining in TCPOBOP-treated mice. (G) The expression of KI67 was measured by KI67 staining, the number of KI67 positive cells was increased in PV area after treated by TCPOBOP ( $n=5$ per group). Scale bar $=50 \mu \mathrm{m}$. (H) Quantification of KI67 staining in TCPOBOP-treated mice. Data are presented as mean $\pm S . D .{ }^{*} P<0.05$, significantly different from Vehicle $5 \mathrm{~d}$; ${ }^{\#} P<0.05$, significantly different from Vehicle $10 \mathrm{~d}$; Student's $t$ test.

FIGURE 2 CAR activation promotes liver regeneration and facilitates the restore of liver weight after partial hepatectomy. (A) Mice after partial hepatectomy were treated with TCPOBOP for 2 days or 5 days. (B) The liver-to-body weight ratio was much higher in TCPOBOP-treated group ( $n=5$ per group). (C) Liver morphology suggested an increase of liver size. (D) H\&E staining suggested that no liver injury occurred $(n$ $=5$ per group). Scale bar $=50 \mu \mathrm{m}$. (E) Hepatocytes size was quantified by CTNNB1 staining which showed hepatocytes hypertrophy around CV area ( $n=5$ per group). Scale bar $=50 \mu \mathrm{m}$. (F) Quantification of CTNNB1 staining in TCPOBOP-treated mice after PHx. (G) The number of KI67 positive cells significantly was increased around PV area which indicated an increase of proliferative response ( $n=5$ per group). Scale bar $=50 \mu \mathrm{m}$. (H) Quantification of KI67 staining in TCPOBOP-treated mice after PHx. Data are presented as mean $\pm S . D .{ }^{*} P<0.05$, significantly different from $\mathrm{PHx} 2 \mathrm{~d}+$ Vehicle; ${ }^{*} P<0.05$, significantly different from PHx 5d+Vehicle; Student's ttest.

FIGURE 3 YAP participates in CAR-induced hepatomegaly and liver regeneration. (A) Total YAP, nuclear YAP and YAP downstream targets were all upregulated, while the cytoplasmic p-YAP was downregulated, which indicated the activation of YAP pathway. Proliferation-related proteins were also upregulated after treated by TCPOBOP ( $n=5$ per group). (B) In PHx model, total YAP, nuclear YAP and YAP downstream targets CTGF, ANKRD1 were upregulated, cytoplasmic p-YAP was downregulated. Meanwhile proliferationrelated proteins such as CCND1 and CCNA1 were upregulated ( $n=5$ per group). (C) The intensity of nuclear YAP was much higher after treated by CITCO in HepG2 cells, which indicated that CAR activation promotes the nuclear translocation of YAP ( $n=6$ per group). (D) Co-immunoprecipitation results suggested the potential protein-protein interaction between CAR and YAP ( $n=6$ per group). Scale bar $=20 \mu \mathrm{m}$.

FIGURE 4 CAR-induced hepatomegaly is partially via YAP pathway. (A) Wildtype or $\mathrm{Yap}^{-/-}$mice were treated by TCPOBOP for 10 days. (B) The liver-to-body weight ratio in TCPOBOP-treated $\mathrm{Yap}^{-/-}$mice was lower than TCPOBOP-treated wildtype mice, but was still much higher than vehicle group of $\mathrm{Yap}^{-/-}$ mice $\left(n=5\right.$ per group). (C) Liver morphology suggested that liver size was increased even in $\mathrm{ap}^{-/-}$mice. (D) H\&E staining suggested that no liver injury occurred ( $n=5$ per group). Scale bar $=50 \mu \mathrm{m}$. (E) CTNNB1 staining showed that there was still a slight increase of hepatocytes size around CV area in $\mathrm{Yap}^{-/-}$mice $(n$ $=5$ per group). Scale bar $=50 \mu \mathrm{m}$. (F) Quantification of CTNNB1 staining in wildtype or Yap $^{-/-}$mice. (G) The number of KI67 positive cells still was increased in $\mathrm{Yap}^{-/-}$mice around PV area ( $n=5$ per group). Scale bar $=50 \mu \mathrm{m}$. (H) Quantification of KI67 staining in wildtype or $\mathrm{Yap}^{-/-}$mice. Data are presented as mean \pm S.D. ${ }^{*} P<0.05$, significantly different from $\mathrm{Yap}^{+/+}+$Vehicle; ${ }^{*} P<0.05$, significantly different from $\mathrm{Yap}^{-/-}$ + Vehicle ${ }^{\S} P<0.05$, significantly different from $\mathrm{Yap}^{+/+}+$TCPOBOP; Student's $t$ test.

FIGURE 5 Other factors are involved in CAR-induced hepatomegaly. (A) The mRNA level of other related genes in wildtype mice treated by TCPOBOP or corn oil for 5 days or 10 days ( $n=5$ per group). (B) The mRNA level of other related genes in wildtype or $\mathrm{Yap}^{-/}$mice $(n=5$ per group). (C) The protein levels of C-Myc, FOXM1 and MET were upregulated after treated by TCPOBOP for 5 days or 10 days $(n=5$ per group). (D) The protein levels of C-Myc, FOXM1, CTNNB1 and MET were upregulated after treated by TCPOBOP in $\mathrm{Yap}^{-/-}$mice $\left(n=5\right.$ per group). Data are presented as mean $\pm S . D .{ }^{*} P<0.05$, significantly different from Vehicle $5 \mathrm{~d}$; $\# P<0.05$, significantly different from Vehicle $10 \mathrm{~d} ;{ }^{\S} P<0.05$, significantly different from $\mathrm{Yap}^{+/+}+$Vehicle $^{\$} P<0.05$, significantly different from $\mathrm{Yap}^{-/-}+$Vehicle; Student's $t$ test. 
A

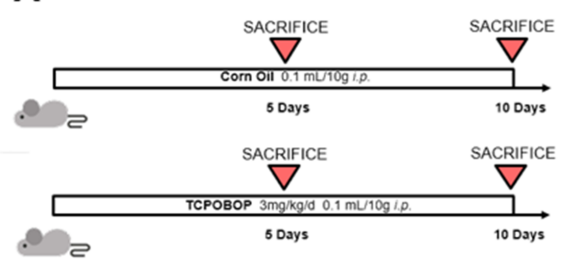

C

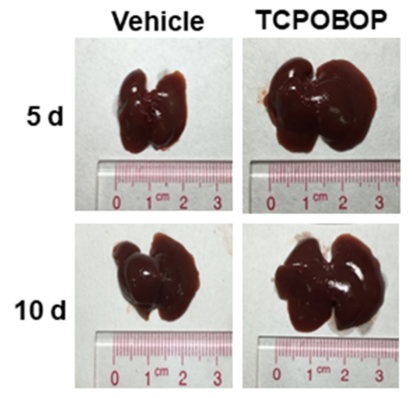

E Vehicle CTNNB1 TCPOBOP

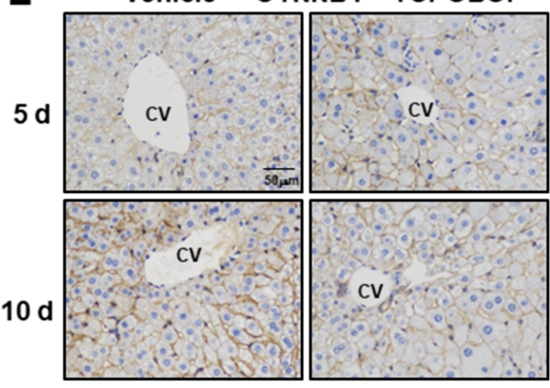

G $\quad$ Vehicle $\quad$ KI67 $\quad$ TCPOBOP

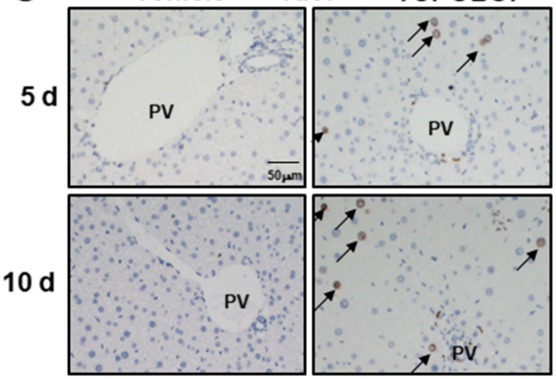

B

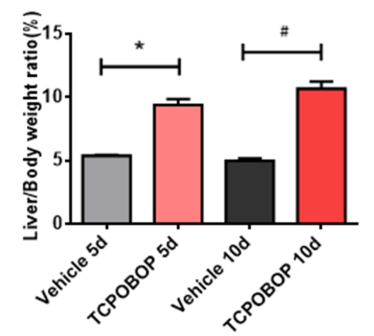

D Vehicle H\&E TCPOBOP

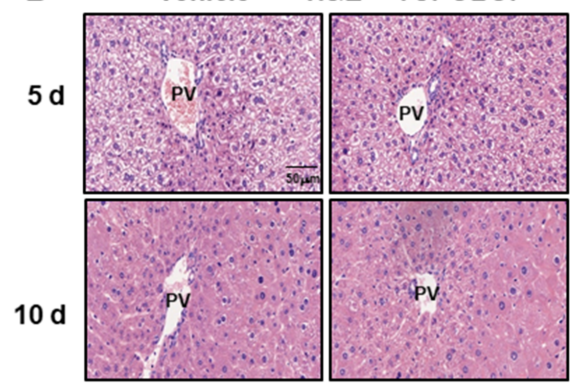

F

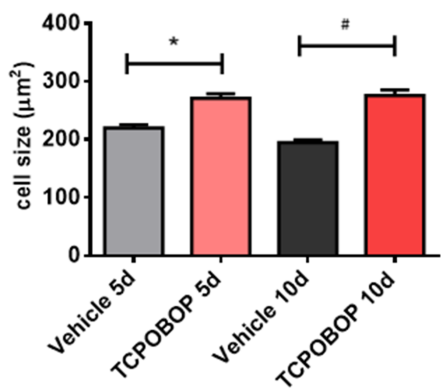

$\mathrm{H}$

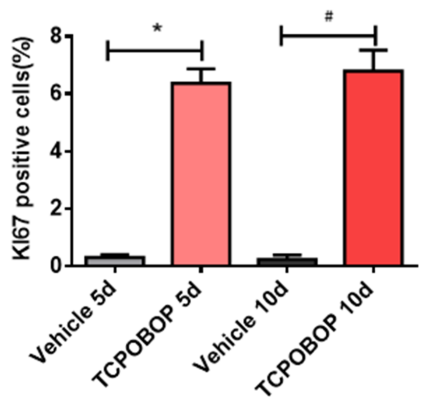


A
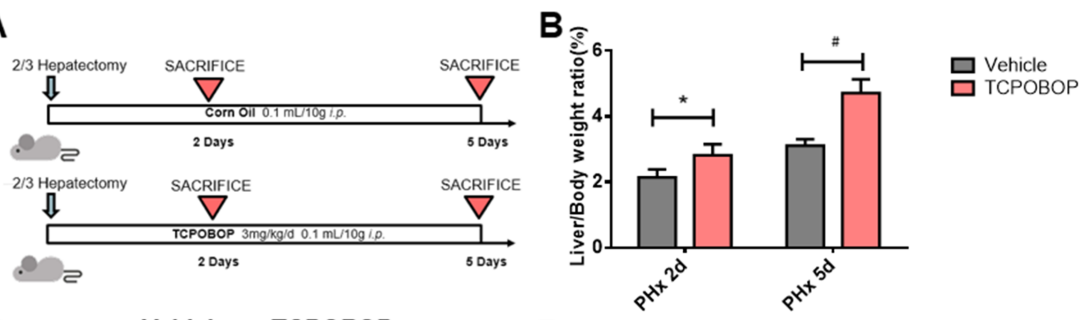

C
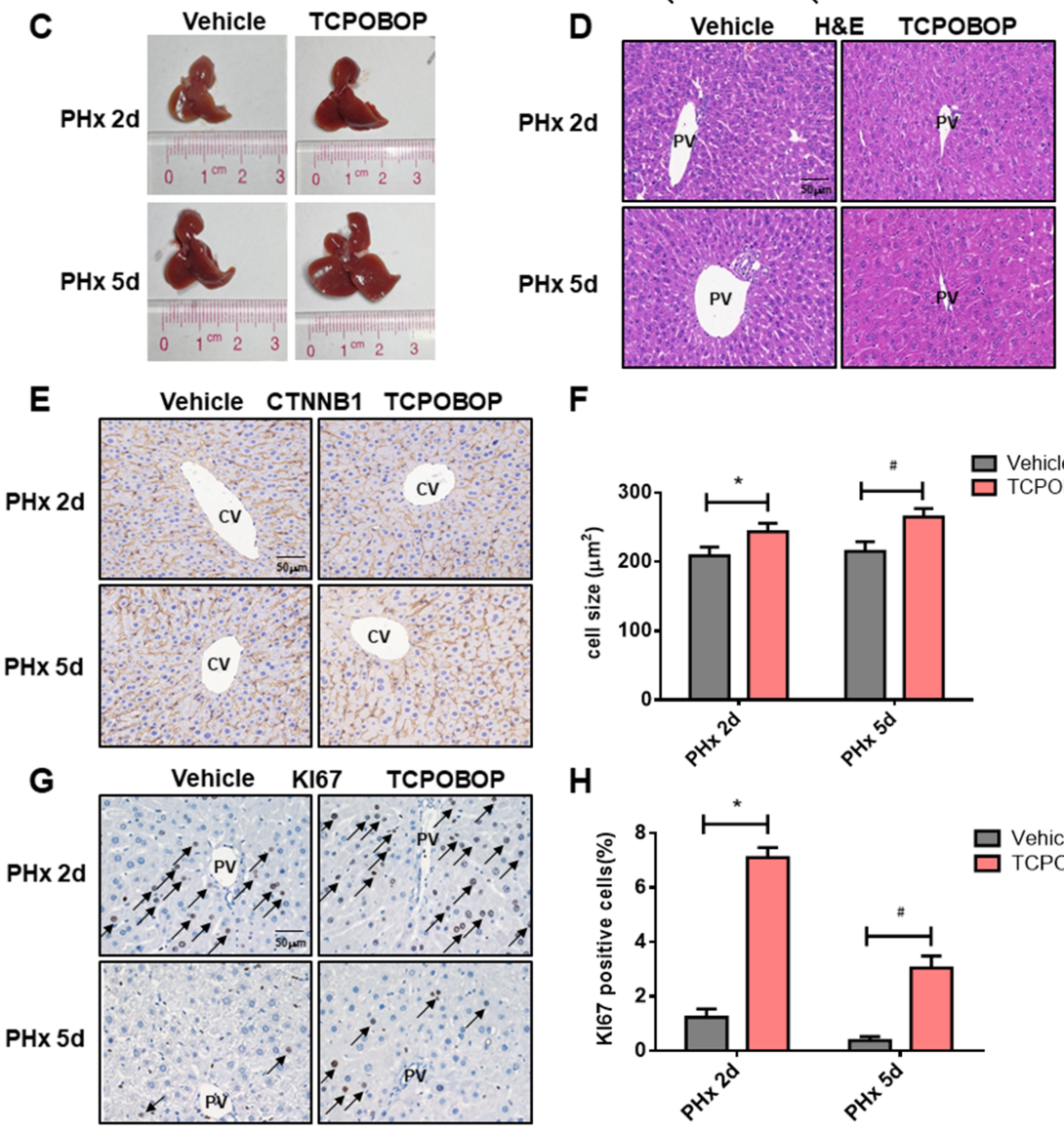

F
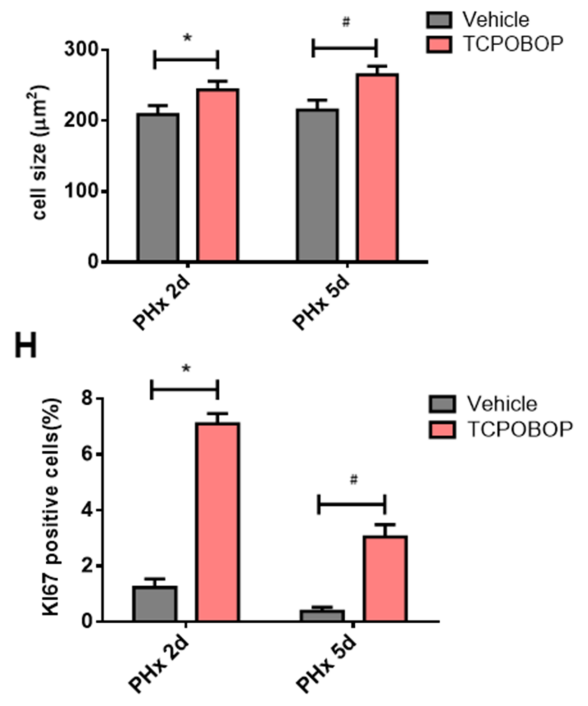
A

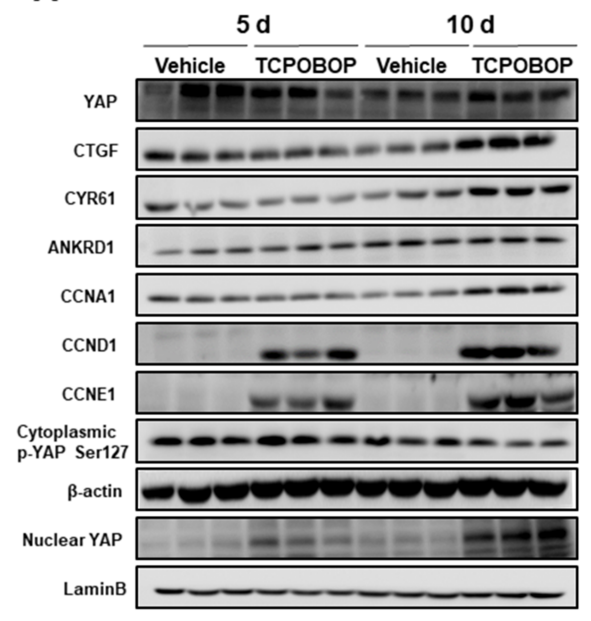

B

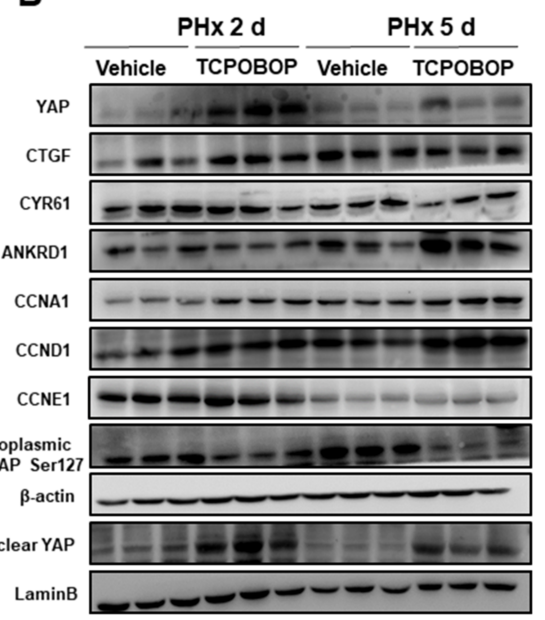

c

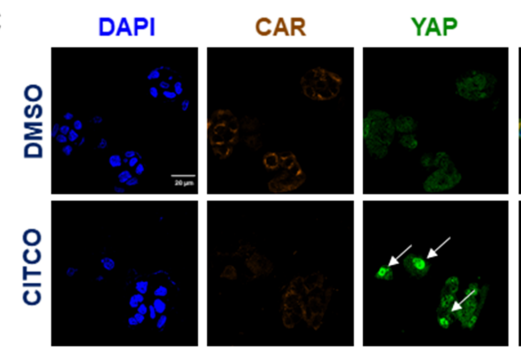

Merge

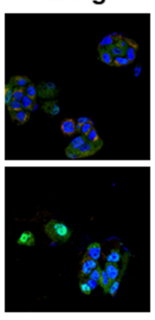

D

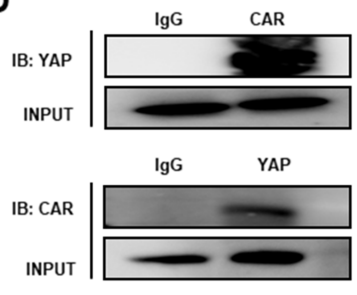



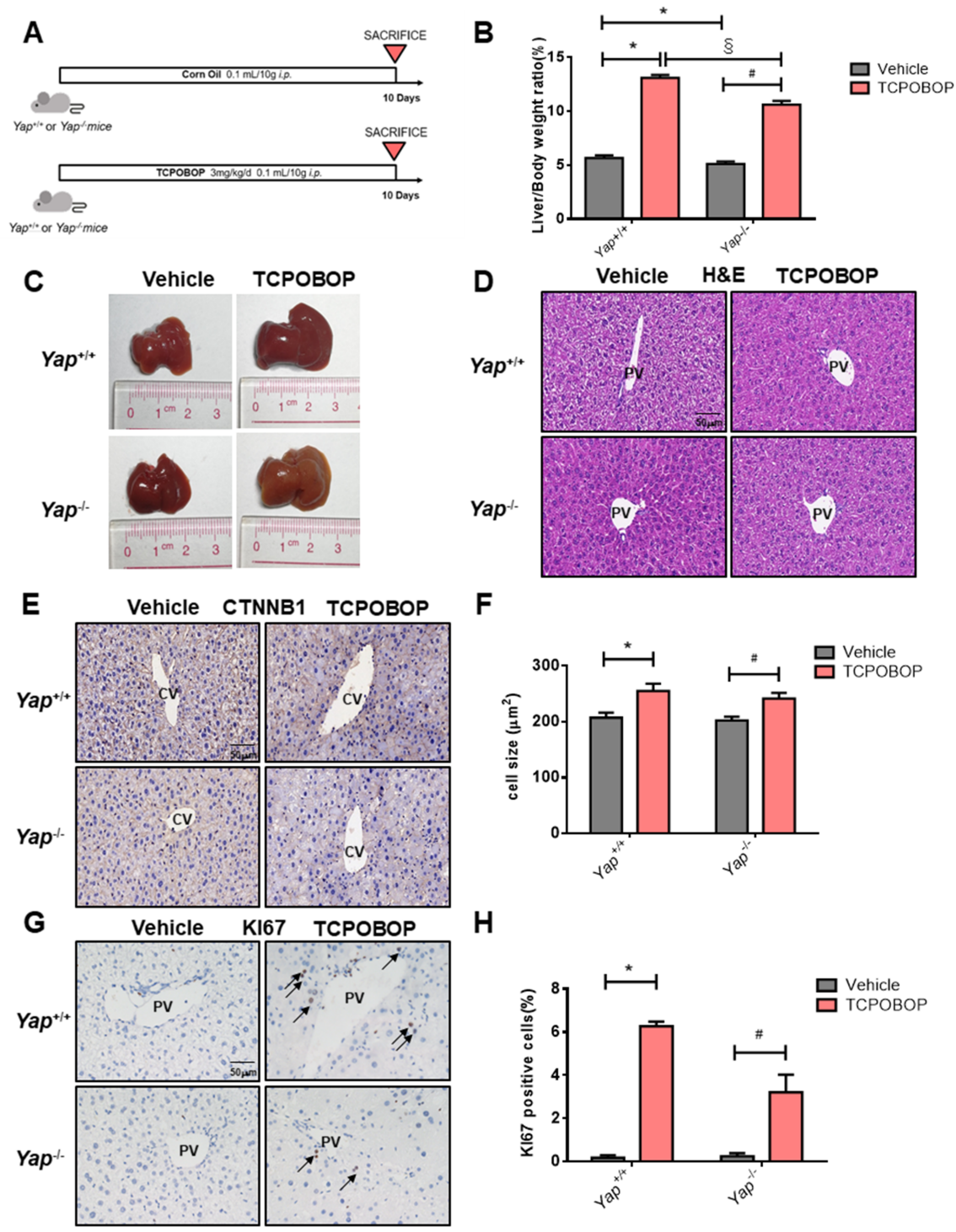

F

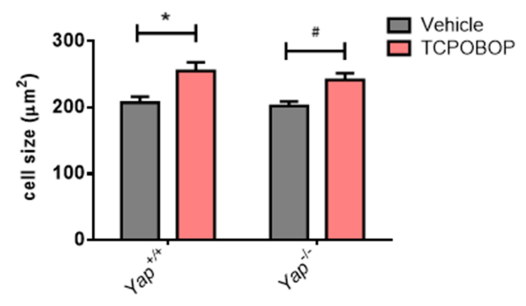

H

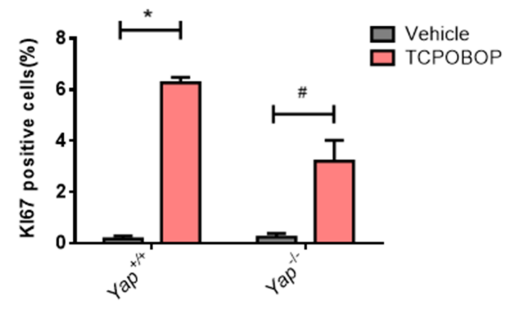


A

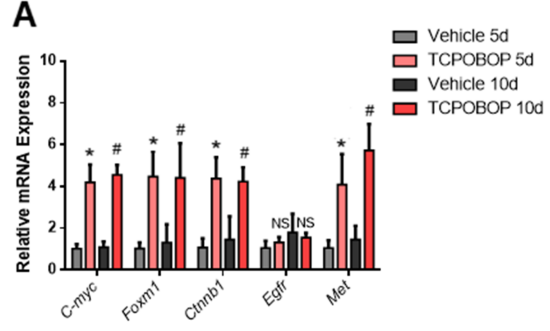

C

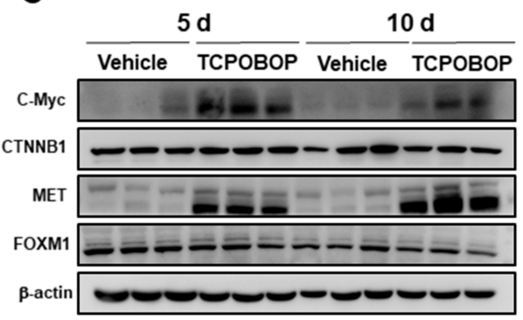

$\mathbf{E}$

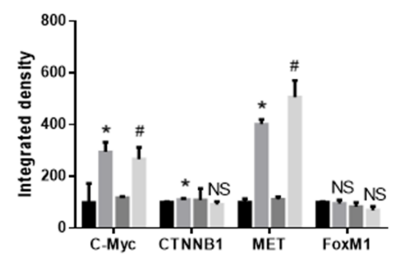

B

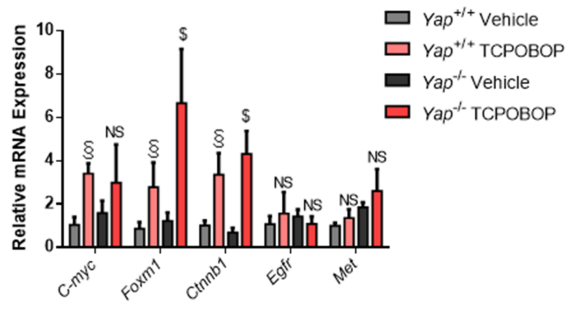

D
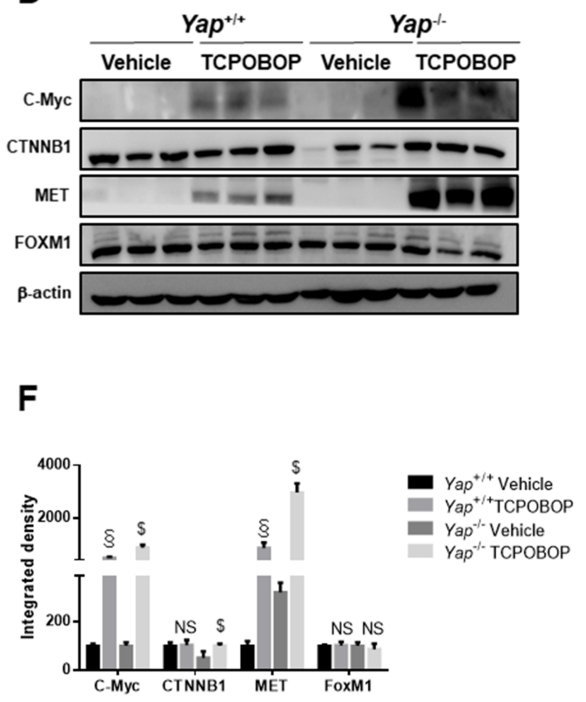

\section{Hosted file}

Table. docx available at https://authorea.com/users/308299/articles/446653-constitutive-androstanereceptor-promoted-hepatomegaly-and-liver-regeneration-is-partially-via-yes-associated-proteinactivation 